Journal of Applied Analysis

Vol. 11, No. 2 (2005), pp. 225-245

\title{
OPTIMALITY CONDITIONS AND DUALITY FOR MULTIOBJECTIVE CONTROL PROBLEMS
}

\author{
T. R. GULATI, I. HUSAIN and A. AHMED \\ Received June 25, 2002 and, in revised form, July 27, 2004
}

\begin{abstract}
Fritz John and Kuhn-Tucker type necessary optimality conditions for a Pareto optimal (efficient) solution of a multiobjective control problem are obtained by first reducing the multiobjective control problem to a system of single objective control problems, and then using already established optimality conditions. As an application of Kuhn-Tucker type optimality conditions, Wolfe and Mond-Weir type dual multiobjective control problems are formulated and usual duality results are established under invexity/generalized invexity, relating properly efficient solutions of the primal and dual problems. Wolfe and Mond-Weir type dual multiobjective control problems with free boundary conditions are also presented.
\end{abstract}

\section{Introduction}

Optimality conditions and duality constitute an essential part of study of mathematical programming in the sense that these lay down the foundation of algorithms for a solution of an optimization problem. In this paper we

2000 Mathematics Subject Classification. 90C46, 90C29.

Key words and phrases. Pareto optimality, efficiency, proper efficiency, multiobjective control problems, duality, free boundary conditions, static problems.

ISSN 1425-6908（C) Heldermann Verlag. 
obtain Fritz John and Kuhn-Tucker type necessary optimality conditions and duality for a multiobjective control problem. We derive these two sets of optimality conditions by reducing the multiobjective control problem to a system of single objective control problems and then using the Fritz John and Kuhn-Tucker type necessary optimality conditions for a single objective control problem, obtained by Chandra, Craven and Husain [1]. As an application of Kuhn-Tucker type optimality conditions, two distinct duals to a multiobjective control problem are formulated and appropriate duality theorems are proved under the hypotheses of invexity/generalized invexity, relating properly efficient solutions of the primal and dual control problems. A pair of dual multiobjective control problems with free boundary conditions is also presented.

\section{Preliminaries and the multiobjective control problem}

Let $f(t, x, u)$, where $t \in I=[a, b] \subset \mathbb{R}, x(t) \in \mathbb{R}^{n}$ and $u(t) \in \mathbb{R}^{r}$, be a $p$-dimensional vector function. Here $t$ is the independent variable and the control variable $u(t)$ is related to the state variable $x(t)$ via the state equation $\dot{x}=h(t, x, u)$, where $\operatorname{dot}(\cdot)$ denotes derivative with respect to $t$. If $\lambda \in \mathbb{R}^{p}$, then $\lambda^{T} f(x)$ is a scalar valued function. Let $\left(\lambda^{T} f\right)_{x}$ and $\left(\lambda^{T} f\right)_{u}$ denote gradient (column) vectors with respect to $x$ and $u$ respectively. Subsequently, $\left(\lambda^{T} f\right)_{x x},\left(\lambda^{T} f\right)_{x u},\left(\lambda^{T} f\right)_{u x}$ and $\left(\lambda^{T} f\right)_{u u}$ denote respectively the $n \times n, n \times r, r \times n$ and $r \times r$ matrices of second order partial derivatives. The gradient of $m$-dimensional vector function $g$ and $n$-dimensional vector function $h$ with respect to $x$ are respectively the $n \times m$ and $n \times n$ matrices. Gradients with respect to $u$ are similarly defined. If $y$ and $z$ are in $\mathbb{R}^{n}$, we denote $y \geqq z \Leftrightarrow y_{i} \geqq z_{i} \quad(i=1, \ldots, n) ; \quad y \geq z \Leftrightarrow(y \geqq z$ and $y \neq z)$; $y>z \Leftrightarrow y_{i}>z_{i}(i=1, \ldots, n)$.

We consider the following multiobjective control problem:

Primal (CP): Minimize

$$
\int_{a}^{b} f(t, x, u) d t=\left[\int_{a}^{b} f^{1}(t, x, u) d t, \cdots, \int_{a}^{b} f^{p}(t, x, u) d t\right]
$$

subject to

$$
\begin{gathered}
x(a)=\alpha, \quad x(b)=\beta, \\
\dot{x}(t)=h(t, x, u), t \in I, \\
g(t, x, u) \geqq 0, t \in I,
\end{gathered}
$$

where $f, g$ and $h$ are twice continuously differentiable functions. We shall use $K$ for the set of all feasible solutions of (CP).

We need the following definitions. 
Definition $2.1([3])$. A point $\left(x^{0}, u^{0}\right) \in K$ is said to be efficient for (CP) if there exists no $(x, u) \in K$ such that

$$
\int_{a}^{b} f(t, x, u) d t \leq \int_{a}^{b} f\left(t, x^{0}, u^{0}\right) d t
$$

An efficient solution is also known as noninferior or nondominated or Pareto optimal solution.

The point $\left(x^{0}, u^{0}\right) \in K$ is said to be properly efficient if it is efficient for (CP) and if there exists a scalar $M>0$ such that, for each $(x, u) \in K$ and $i \in\{1,2, \cdots, p\}$ satisfying $\int_{a}^{b} f^{i}(t, x, u) d t<\int_{a}^{b} f^{i}\left(t, x^{0}, u^{0}\right) d t$, we have

$$
\begin{aligned}
& \int_{a}^{b} f^{i}\left(t, x^{0}, u^{0}\right) d t-\int_{a}^{b} f^{i}(t, x, u) d t \\
& \leqq M\left(\int_{a}^{b} f^{j}(t, x, u) d t-\int_{a}^{b} f^{j}\left(t, x^{0}, u^{0}\right) d t\right)
\end{aligned}
$$

for some $j \in\{1,2, \cdots, p\} \backslash\{i\}$ such that

$$
\int_{a}^{b} f^{j}(t, x, u) d t>\int_{a}^{b} f^{j}\left(t, x^{0}, u^{0}\right) d t
$$

Definition $2.2([8])$. If there exist a vector functions $\eta(t, x, w, \dot{x}, \dot{w}, u, v) \in$ $\mathbb{R}^{n}$ with $\eta=0$ at $t$ if $x(t)=w(t)$ and $\xi(t, x, w, \dot{x}, \dot{w}, u, v) \in \mathbb{R}^{r}$ such that for the scalar function $\theta(t, x, \dot{x}, u)$, the functional $\Theta(x, \dot{x}, u)=\int_{a}^{b} \theta(t, x, \dot{x}, u) d t$ satisfies

$$
\begin{aligned}
& \Theta(x, \dot{x}, u)-\Theta(w, \dot{w}, v) \\
& \geqq \int_{a}^{b}\left[\eta^{T} \theta_{x}(t, w, \dot{w}, v)+\left(\frac{d \eta}{d t}\right)^{T} \theta_{\dot{x}}(t, w, \dot{w}, v)+\xi^{T} \theta_{u}(t, w, \dot{w}, v)\right] d t,
\end{aligned}
$$

then $\Theta$ is said to be invex in $x, \dot{x}$ and $u$ with respect to $\eta$ and $\xi$ on $[a, b]$.

The functional $\Theta$ is said to be pseudo-invex in $x, \dot{x}$ and $u$ with respect to $\eta$ and $\xi$ on $[a, b]$ if

$$
\begin{aligned}
\int_{a}^{b}\left[\eta^{T} \theta_{x}(t, w, \dot{w}, v)\right. & \left.+\left(\frac{d \eta}{d t}\right)^{T} \theta_{\dot{x}}(t, w, \dot{w}, v)+\xi^{T} \theta_{u}(t, w, \dot{w}, v)\right] d t \geqq 0 \\
& \Rightarrow \Theta(x, \dot{x}, u) \geqq \Theta(w, \dot{w}, v) .
\end{aligned}
$$

Further $\Theta$ is said to be quasi-invex in $x, \dot{x}$ and $u$ with respect to $\eta$ and $\xi$ on $[a, b]$ if

$$
\Theta(x, \dot{x}, u) \leqq \Theta(w, \dot{w}, v)
$$




$$
\Rightarrow \int_{a}^{b}\left[\eta^{T} \theta_{x}(t, w, \dot{w}, v)+\left(\frac{d \eta}{d t}\right)^{T} \theta_{\dot{x}}(t, w, \dot{w}, v)+\xi^{T} \theta_{u}(t, w, \dot{w}, v)\right] d t \leqq 0 .
$$

\section{Necessary optimality conditions}

In this section, Fritz John and Kuhn-Tucker type necessary Pareto optimality conditions are derived by reducing the multiobjective control problem stated in Section 1 to a system of single objective control problems and then using the known optimality results for each problem. Here the approach of derivation of these optimality conditions is very much in the spirit of [4].

The following proposition establishes the linkage between multiobjective and single objective control problems. Its proof follows on the lines of Lemma 3.1 in Kanniappan [6].

Proposition 3.1. A point $\left(x^{0}, u^{0}\right)$ is an efficient solution of $(C P)$ if and only if for each $i \in\{1,2, \cdots, p\},\left(x^{0}, u^{0}\right)$ is optimal to the single objective control problem

$(\mathbf{C P})^{\mathbf{i}}$ : Minimize $\int_{a}^{b} f^{i}(t, x, u) d t \quad$ subject to

$$
\begin{gathered}
x(a)=\alpha, \quad x(b)=\beta, \\
\dot{x}(t)=h(t, x, u), t \in I, \\
g(t, x, u) \geqq 0, t \in I, \\
\int_{a}^{b} f^{k}(t, x, u) d t \leqq \int_{a}^{b} f^{k}\left(t, x^{0}, u^{0}\right) d t, k \neq i .
\end{gathered}
$$

Consider the following single objective control problem, studied by Mond and Hanson [7] and Chandra, Craven and Husain [1].

(CP) : Minimize $\int_{a}^{b} \phi(t, x, u) d t \quad$ subject to

$$
\begin{gathered}
x(a)=\alpha, \quad x(b)=\beta, \\
\dot{x}(t)=h(t, x, u), t \in I, \\
g(t, x, u) \geqq 0, t \in I .
\end{gathered}
$$

Here $\phi: I \times X \times U \rightarrow \mathbb{R}$ is twice continuously differentiable.

Following Craven [2], the differential equation (2) for $x(t)$ with initial condition expressed as

$$
x(t)=x(a)+\int_{a}^{t} h(s, x(s), u(s)) d s, t \in I,
$$

may then be written as

$$
D x=H(x, u),
$$


where the map $H: X \times U \rightarrow C\left(I, \mathbb{R}^{n}\right)$ is defined by

$$
H(x, u)(t)=h(t, x(t), u(t))
$$

in which

(i) $C\left(I, \mathbb{R}^{n}\right)$ is the space of continuous functions from $I$ into $\mathbb{R}^{n}$, with the norm, $\|\phi(t)\|=\sup _{t \in I}|\phi(t)|$,

(ii) $X$ is the space of continuous state functions $x: I \rightarrow \mathbb{R}^{n}$ such that $x(a)=\alpha, x(b)=\beta$, and

(iii) $U$ is the space of piecewise continuous control functions $u: I \rightarrow \mathbb{R}^{r}$ with uniform norm $\|\cdot\|_{\infty}$.

In the following Fritz John type optimality theorem, established by Chandra, Craven and Husain [1], the Fréchet derivative of $Q(x, u)=$ $D x-H(x, u)$, i.e.,

$$
Q^{\prime}=Q^{\prime}\left(x^{0}, u^{0}\right)=\left[D-H_{x}\left(x^{0}, u^{0}\right)-H_{u}\left(x^{0}, u^{0}\right)\right]
$$

is needed to be surjective in order to make the equality constraints locally solvable.

Theorem $3.1([1])$. If $\left(x^{0}, u^{0}\right)$ is an optimal solution of $(C P)_{\phi}$ and the Fréchet derivative $Q^{\prime}$ is surjective, then there exist Lagrange multipliers $\zeta \in \mathbb{R}$ and piecewise smooth functions $z: I \rightarrow \mathbb{R}^{n}$ and $y: I \rightarrow \mathbb{R}^{m}$ such that for all $t \in I$,

$$
\begin{aligned}
& \zeta \phi_{x}\left(t, x^{0}(t), u^{0}(t)\right)-z(t)^{T} h_{x}\left(t, x^{0}(t), u^{0}(t)\right)-y(t)^{T} g_{x}\left(t, x^{0}(t), u^{0}(t)\right)=\dot{z}(t), \\
& \zeta \phi_{u}\left(t, x^{0}(t), u^{0}(t)\right)-z(t)^{T} h_{u}\left(t, x^{0}(t), u^{0}(t)\right)-y(t)^{T} g_{u}\left(t, x^{0}(t), u^{0}(t)\right)=0, \\
& y(t)^{T} g\left(t, x^{0}(t), u^{0}(t)\right)=0, \\
& y(t) \geqq 0, \\
& \zeta \geqq 0, \\
& (\zeta, z(t), y(t)) \neq 0 .
\end{aligned}
$$

Theorem 3.2 (Fritz John type necessary conditions). If $\left(x^{0}, u^{0}\right)$ is an efficient solution of $(C P)$ and the Fréchet derivative $Q^{\prime}$ is surjective, then there exist Lagrangian multipliers $\lambda=\left(\lambda^{1}, \lambda^{2}, \cdots, \lambda^{p}\right) \in \mathbb{R}^{p}$ and piecewise smooth functions $z: I \rightarrow \mathbb{R}^{n}$ and $y: I \rightarrow \mathbb{R}^{m}$ such that

$$
\begin{array}{r}
\lambda^{T} f_{x}\left(t, x^{0}(t), u^{0}(t)\right)-z(t)^{T} h_{x}\left(t, x^{0}(t), u^{0}(t)\right)-y(t)^{T} g_{x}\left(t, x^{0}(t), u^{0}(t)\right)=\dot{z}(t), \\
t \in I, \\
\lambda^{T} f_{u}\left(t, x^{0}(t), u^{0}(t)\right)-z(t)^{T} h_{u}\left(t, x^{0}(t), u^{0}(t)\right)-y(t)^{T} g_{u}\left(t, x^{0}(t), u^{0}(t)\right)=0, \\
t \in I, \\
y(t)^{T} g\left(t, x^{0}(t), u^{0}(t)\right)=0, t \in I,
\end{array}
$$


$y(t) \geqq 0, t \in I$,

$\lambda \geqq 0$,

$(\lambda, z(t), y(t)) \neq 0, t \in I$.

Proof. Suppose $\left(x^{0}, u^{0}\right)$ is efficient for $(\mathrm{CP})$. By Proposition 3.1, $\left(x^{0}, u^{0}\right)$ is optimal for the following single objective control problem

$(\mathbf{C P})^{\mathbf{1}}$ : Minimize $\quad \int_{a}^{b} f^{1}(t, x, u) d t \quad$ subject to

$$
\begin{gathered}
x(a)=\alpha, \quad x(b)=\beta, \\
\dot{x}^{r}(t)=h^{r}(t, x, u), t \in I, r \in\{1,2, \cdots, n\}, \\
g^{j}(t, x, u) \geqq 0, t \in I, j \in\{1,2, \cdots, m\}, \\
\int_{a}^{b} f^{i}(t, x, u) d t \leqq \int_{a}^{b} f^{i}\left(t, x^{0}, u^{0}\right) d t, i \in\{2,3, \cdots, p\} .
\end{gathered}
$$

Hence by Theorem 3.1 there exist $\lambda=\left(\lambda^{1}, \lambda^{2}, \cdots, \lambda^{p}\right) \in \mathbb{R}_{+}^{p}$, piecewise smooth functions $z: I \rightarrow \mathbb{R}^{n}$ with $z=\left(z^{1}, z^{2}, \cdots, z^{n}\right)^{T}$ and $y: I \rightarrow \mathbb{R}_{+}^{m}$ with $y=\left(y^{1}, y^{2}, \cdots, y^{m}\right)^{T}$ such that

$$
\begin{aligned}
& \lambda^{1} f_{x}^{1}+\sum_{i=2}^{p} \lambda^{i} f_{x}^{i}-\sum_{r=1}^{n} z^{r}(t) h_{x}^{r}-\sum_{j=1}^{m} y^{j}(t) g_{x}^{j}=\dot{z}(t), t \in I \\
& \lambda^{1} f_{u}^{1}+\sum_{i=2}^{p} \lambda^{i} f_{u}^{i}-\sum_{r=1}^{n} z^{r}(t) h_{u}^{r}-\sum_{j=1}^{m} y^{j}(t) g_{u}^{j}=0, t \in I, \\
& \lambda^{i}\left[\int_{a}^{b} f^{i}(t, x, u) d t-\int_{a}^{b} f^{i}\left(t, x^{0}, u^{0}\right) d t\right]=0, i \in\{2,3, \cdots, p\} \\
& y(t)^{T} g\left(t, x^{0}, u^{0}\right)=0, t \in I \\
& (\lambda, z(t), y(t)) \neq 0, t \in I . \\
&
\end{aligned}
$$

That is,

$$
\begin{aligned}
& \lambda^{T} f_{x}-z(t)^{T} h_{x}-y(t)^{T} g_{x}=\dot{z}(t), t \in I, \\
& \lambda^{T} f_{u}-z(t)^{T} h_{u}-y(t)^{T} g_{u}=0, t \in I, \\
& y(t)^{T} g\left(t, x^{0}, u^{0}\right)=0, t \in I, \\
& y(t) \geqq 0, t \in I, \\
& \lambda \geqq 0, \\
& (\lambda, z(t), y(t)) \neq 0, t \in I .
\end{aligned}
$$

This completes the proof. 
We shall use the following Kuhn-Tucker type necessary optimality conditions for $(\mathrm{CP})_{\phi}$, obtained by Chandra, Craven and Husain [1]. Theorem 3.1 gives Kuhn-Tucker type necessary optimality conditions, if $\zeta=1$. Then $\left(x^{0}, u^{0}\right)$ will be called normal.

Theorem $3.3([1])$. If $\left(x^{0}, u^{0}\right)$ is an optimal solution for $(C P)_{\phi}$ and is normal, and the Fréchet derivative $Q^{\prime}$ is surjective, then there exist piecewise smooth functions $z: I \rightarrow \mathbb{R}^{n}$ and $y: I \rightarrow \mathbb{R}^{m}$ such that

$$
\begin{array}{r}
\phi_{x}\left(t, x^{0}(t), u^{0}(t)\right)-z(t)^{T} h_{x}\left(t, x^{0}(t), u^{0}(t)\right)-y(t)^{T} g_{x}\left(t, x^{0}(t), u^{0}(t)\right)=\dot{z}(t), \\
t \in I, \\
\phi_{u}\left(t, x^{0}(t), u^{0}(t)\right)-z(t)^{T} h_{u}\left(t, x^{0}(t), u^{0}(t)\right)-y(t)^{T} g_{u}\left(t, x^{0}(t), u^{0}(t)\right)=0, \\
t \in I,
\end{array}
$$

$y(t)^{T} g\left(t, x^{0}(t), u^{0}(t)\right)=0, t \in I$,

$y(t) \geqq 0, t \in I$.

In the following analysis, whenever we assume that the solution $\left(x^{0}, u^{0}\right)$ of $(\mathrm{CP})$ is normal, we mean that it is normal to $(\mathrm{CP})^{i}$, for each $i \in$ $\{1,2, \cdots, p\}$. Also, the gradients $f_{x}, f_{u}$ etc. are at $\left(t, x^{0}(t), u^{0}(t)\right)$.

Theorem 3.4 (Kuhn-Tucker type necessary conditions). If $\left(x^{0}, u^{0}\right)$ is efficient for $(C P)$ and is normal, and the Fréchet derivative $Q^{\prime}$ is surjective, then there exist a vector $\lambda \in \mathbb{R}^{p}$ and piecewise smooth functions $z: I \rightarrow \mathbb{R}^{n}$ and $y: I \rightarrow \mathbb{R}^{m}$ such that

$$
\begin{aligned}
& \lambda^{T} f_{x}-z(t)^{T} h_{x}-y(t)^{T} g_{x}=\dot{z}(t), t \in I, \\
& \lambda^{T} f_{u}-z(t)^{T} h_{u}-y(t)^{T} g_{u}=0, t \in I, \\
& y(t)^{T} g\left(t, x^{0}, u^{0}\right)=0, t \in I, \\
& y(t) \geqq 0, t \in I, \\
& \lambda>0, \quad \lambda^{T} e=1,
\end{aligned}
$$

where $e=(1,1, \cdots, 1)^{T} \in \mathbb{R}^{p}$.

Proof. Since $\left(x^{0}, u^{0}\right)$ is efficient for (CP), by Proposition 3.1, for each $i \in$ $\{1,2, \cdots, p\},\left(x^{0}, u^{0}\right)$ is optimal to

$(\mathbf{C P})^{\mathbf{i}}$ : Minimize $\quad \int_{a}^{b} f^{i}(t, x, u) d t \quad$ subject to

$$
\begin{gathered}
x(a)=\alpha, \quad x(b)=\beta, \\
\dot{x}^{r}(t)=h^{r}(t, x, u), t \in I, r \in\{1,2, \cdots, n\}, \\
g^{j}(t, x, u) \geqq 0, t \in I, j \in\{1,2, \cdots, m\},
\end{gathered}
$$




$$
\int_{a}^{b} f^{k}(t, x, u) d t \leqq \int_{a}^{b} f^{k}\left(t, x^{0}, u^{0}\right) d t, k \neq i
$$

Then, by Theorem 3.3, there exist Lagrange multipliers $a^{k i}, k=$ $1,2, \cdots, p, k \neq i ; b^{r i}(t), i=1,2, \cdots, n$ and $c^{j i}(t), j=1,2, \cdots, m$ such that

$$
\begin{aligned}
& f_{x}^{i}+\sum_{\substack{k=1 \\
k \neq i}}^{p} a^{k i} f_{x}^{k}-\sum_{r=1}^{n} b^{r i}(t) h_{x}^{r}-\sum_{j=1}^{m} c^{j i}(t) g_{x}^{j}=\left(\dot{b}^{1 i}(t), \cdots, \dot{b}^{n i}(t)\right)^{T}, \\
& t \in I, \\
& f_{u}^{i}+\sum_{\substack{k=1 \\
k \neq i}}^{p} a^{k i} f_{u}^{k}-\sum_{r=1}^{n} b^{r i}(t) h_{u}^{r}-\sum_{j=1}^{m} c^{j i}(t) g_{u}^{j}=0, t \in I, \\
& c^{j i}(t) g^{j}\left(t, x^{0}, u^{0}\right)=0, j \in\{1,2, \cdots, m\} \text { and } t \in I .
\end{aligned}
$$

Setting $a^{i i}=1$ for $i=1,2, \cdots, p$, equations (4) and (5) can be written as

$$
\begin{aligned}
& \sum_{k=1}^{p} a^{k i} f_{x}^{k}-\sum_{r=1}^{n} b^{r i}(t) h_{x}^{r}-\sum_{j=1}^{m} c^{j i}(t) g_{x}^{j}=\left(\dot{b}^{1 i}(t), \cdots, \dot{b}^{n i}(t)\right)^{T}, t \in I, \\
& \sum_{k=1}^{p} a^{k i} f_{u}^{k}-\sum_{r=1}^{n} b^{r i}(t) h_{u}^{r}-\sum_{j=1}^{m} c^{j i}(t) g_{u}^{j}=0, t \in I .
\end{aligned}
$$

Adding these equations for all $i$, we get

$$
\begin{aligned}
& \sum_{k=1}^{p}\left(a^{k 1}+a^{k 2}+\cdots+a^{k p}\right) f_{x}^{k}-\sum_{r=1}^{n}\left(b^{r 1}(t)+b^{r 2}(t)+\cdots+b^{r p}(t)\right) h_{x}^{r} \\
& -\sum_{j=1}^{m}\left(c^{j 1}(t)+c^{j 2}(t)+\cdots+c^{j p}(t)\right) g_{x}^{j}=\left(\sum_{i=1}^{p} \dot{b}^{1 i}(t), \cdots, \sum_{i=1}^{p} \dot{b}^{n i}(t)\right)^{T}, t \in I
\end{aligned}
$$

and

$$
\begin{aligned}
& \sum_{k=1}^{p}\left(a^{k 1}+a^{k 2}+\cdots+a^{k p}\right) f_{u}^{k}-\sum_{r=1}^{n}\left(b^{r 1}(t)+b^{r 2}(t)+\cdots+b^{r p}(t)\right) h_{u}^{r} \\
& -\sum_{j=1}^{m}\left(c^{j 1}(t)+c^{j 2}(t)+\cdots+c^{j p}(t)\right) g_{u}^{j}=0, t \in I .
\end{aligned}
$$

Now let

$$
a^{k}=\sum_{i=1}^{p} a^{k i}, b^{r}(t)=\sum_{i=1}^{p} b^{r i}(t) \text { and } \mathrm{c}^{\mathrm{j}}(\mathrm{t})=\sum_{\mathrm{i}=1}^{\mathrm{p}} \mathrm{c}^{\mathrm{ji}}(\mathrm{t}) .
$$


Hence there exist $a^{k}>0, k \in\{1,2, \cdots, p\}$ and piecewise smooth $b^{r}(t)$, $r \in\{1,2, \cdots, n\}$ and $c^{j}(t) \geqq 0, j \in\{1,2, \cdots, m\}$ such that

$$
\begin{aligned}
& \sum_{k=1}^{p} a^{k} f_{x}^{k}-\sum_{r=1}^{n} b^{r}(t) h_{x}^{r}-\sum_{j=1}^{m} c^{j}(t) g_{x}^{j}=\left(\dot{b}^{1}(t), \cdots, \dot{b}^{n}(t)\right), t \in I, \\
& \sum_{k=1}^{p} a^{k} f_{u}^{k}-\sum_{r=1}^{n} b^{r}(t) h_{u}^{r}-\sum_{j=1}^{m} c^{j}(t) g_{u}^{j}=0, t \in I, \\
& c^{j}(t) g^{j}\left(t, x^{0}, u^{0}\right)=0, j \in\{1,2, \cdots, m\} \text { and } t \in I .
\end{aligned}
$$

Now let $\alpha=\sum_{k=1}^{p} a^{k}$. Dividing the above equations throughout by $\alpha(>0)$ and by taking

$$
\frac{a^{k}}{\alpha}=\lambda^{k}>0, \quad \frac{b^{r}(t)}{\alpha}=z^{r}(t) \quad \text { and } \quad \frac{c^{j}(t)}{\alpha}=y^{j}(t),
$$

we have,

$$
\begin{aligned}
& \lambda^{T} f_{x}-z(t)^{T} h_{x}-y(t)^{T} g_{x}=\dot{z}(t), t \in I, \\
& \lambda^{T} f_{u}-z(t)^{T} h_{u}-y(t)^{T} g_{u}=0, t \in I, \\
& y(t)^{T} g(t, x, u)=0, t \in I, \\
& y(t) \geqq 0, t \in I, \\
& \lambda>0, \quad \lambda^{T} e=1 .
\end{aligned}
$$

\section{Wolfe type duality}

For the problem $(\mathrm{CP})$, we consider the following Wolfe type dual problem (WCD).

Wolfe Dual (WCD): Maximize

$$
\int_{a}^{b}\left[f(t, x, u)-\left\{z(t)^{T}(h(t, x, u)-\dot{x}(t))+y(t)^{T} g(t, x, u)\right\} e\right] d t
$$

subject to

$$
\begin{aligned}
& x(a)=\alpha, \quad x(b)=\beta, \\
& \lambda^{T} f_{x}(t, x, u)-z(t)^{T} h_{x}(t, x, u)-y(t)^{T} g_{x}(t, x, u)=\dot{z}(t), t \in I, \\
& \lambda^{T} f_{u}(t, x, u)-z(t)^{T} h_{u}(t, x, u)-y(t)^{T} g_{u}(t, x, u)=0, t \in I, \\
& y(t) \geqq 0, t \in I, \\
& \lambda>0, \quad \lambda^{T} e=1 .
\end{aligned}
$$


If $p=1$, the problems (CP) and (WCD) reduce to the pair of single objective dual control problems with invexity, treated by Mond and Smart [8].

Let $\mathrm{F}$ be the feasible region of (WCD). We shall now see that appropriate duality results hold between (CP) and (WCD).

Theorem 4.1 (Weak duality). Let $\left(x^{0}, u^{0}\right) \in K$ and $(x, u, z, y, \lambda) \in F$. If $\int_{a}^{b} \lambda^{T} f d t,-\int_{a}^{b} z(t)^{T}(h-\dot{x}) d t$ and $-\int_{a}^{b} y(t)^{T} g d t$, are all invex with respect to the same vector functions $\eta$ and $\xi$, then

$$
\begin{aligned}
& \int_{a}^{b} f\left(t, x^{0}, u^{0}\right) d t \\
& \not \int_{a}^{b}\left[f(t, x, u)-\left\{z(t)^{T}(h(t, x, u)-\dot{x}(t))+y(t)^{T} g(t, x, u)\right\} e\right] d t .
\end{aligned}
$$

Proof. By invexity of $\int_{a}^{b} \lambda^{T} f(t, x, u) d t$,

$$
\begin{aligned}
& \int_{a}^{b} \lambda^{T} f\left(t, x^{0}, u^{0}\right) d t-\int_{a}^{b} \lambda^{T} f(t, x, u) d t \\
& \geqq \int_{a}^{b}\left\{\eta^{T}\left(\lambda^{T} f\right)_{x}(t, x, u)+\xi^{T}\left(\lambda^{T} f\right)_{u}(t, x, u)\right\} d t \\
& =\int_{a}^{b} \eta^{T}\left\{\dot{z}(t)+\left(z(t)^{T} h\right)_{x}(t, x, u)+\left(y(t)^{T} g\right)_{x}(t, x, u)\right\} d t \\
& +\int_{a}^{b} \xi^{T}\left[\left(z(t)^{T} h\right)_{u}(t, x, u)+\left(y(t)^{T} g\right)_{u}(t, x, u)\right] d t,
\end{aligned}
$$

(using dual constraints (8) and (9))

$$
\begin{aligned}
& =\left.\eta^{T} z(t)\right|_{t=a} ^{t=b}-\int_{a}^{b}\left(\frac{d \eta}{d t}\right)^{T} z(t) d t \\
& +\int_{a}^{b}\left[\eta^{T}\left(z(t)^{T} h\right)_{x}(t, x, u)+\eta^{T}\left(y(t)^{T} g\right)_{x}(t, x, u)\right] d t \\
& +\int_{a}^{b} \xi^{T}\left[\left(z(t)^{T} h\right)_{u}(t, x, u)+\left(y(t)^{T} g\right)_{u}(t, x, u)\right] d t
\end{aligned}
$$

(integrating the first term by parts)

$$
\begin{aligned}
& =\int_{a}^{b}\left[\eta^{T}\left(z(t)^{T} h\right)_{x}(t, x, u)-\left(\frac{d \eta}{d t}\right)^{T} z(t)+\eta^{T}\left(y(t)^{T} g\right)_{x}(t, x, u)\right] d t \\
& +\int_{a}^{b} \xi^{T}\left[\left(z(t)^{T} h\right)_{u}(t, x, u)+\left(y(t)^{T} g\right)_{u}(t, x, u)\right] d t
\end{aligned}
$$

(as fixed conditions give $\eta=0$ at $t=a$ and $t=b$ ) 


$$
\begin{aligned}
& =\int_{a}^{b}\left[\eta^{T}\left(z(t)^{T} h\right)_{x}(t, x, u)-\left(\frac{d \eta}{d t}\right)^{T} z(t)+\xi^{T}\left(z(t)^{T} h\right)_{u}(t, x, u)\right] d t \\
& +\int_{a}^{b}\left[\eta^{T}\left(y(t)^{T} g\right)_{x}(t, x, u)+\xi^{T}\left(y(t)^{T} h\right)_{u}(t, x, u)\right] d t \\
& \geqq \int_{a}^{b} z(t)^{T}\left[\left(h\left(t, x^{0}, u^{0}\right)-\dot{x}^{0}(t)\right)-(h(t, x, u)-\dot{x}(t))\right] d t \\
& +\int_{a}^{b} y(t)^{T}\left[g\left(t, x^{0}, u^{0}\right)-g(t, x, u)\right] d t
\end{aligned}
$$

(by invexity of $-\int_{a}^{b} z(t)^{T}(h-\dot{x}) d t$ and $\left.-\int_{a}^{b} y(t)^{T} g d t\right)$

$$
\geqq \int_{a}^{b}\left[-z(t)^{T}(h(t, x, u)-\dot{x}(t))-y(t)^{T} g(t, x, u)\right] d t
$$

(by (2), (3) and (10)).

Therefore,

$$
\begin{aligned}
& \int_{a}^{b} \lambda^{T} f\left(t, x^{0}, u^{0}\right) d t \\
& \geqq \int_{a}^{b}\left[\lambda^{T} f(t, x, u)-\left\{z(t)^{T}(h(t, x, u)-\dot{x}(t))+y(t)^{T} g(t, x, u)\right\}\right] d t,
\end{aligned}
$$

which, because of $\lambda^{T} e=1$, gives

$$
\begin{aligned}
\lambda^{T}\left(\int_{a}^{b} f\left(t, x^{0}, u^{0}\right) d t\right) & \geqq \lambda^{T} \int_{a}^{b}\left[f(t, x, u)-\left\{z(t)^{T}(h(t, x, u)-\dot{x}(t))\right.\right. \\
& \left.\left.+y(t)^{T} g(t, x, u)\right\} e\right] d t .
\end{aligned}
$$

That is,

$$
\begin{aligned}
& \int_{a}^{b} f\left(t, x^{0}, u^{0}\right) d t \\
& \not \leq \int_{a}^{b}\left[f(t, x, u)-\left\{z(t)^{T}(h(t, x, u)-\dot{x}(t))+y(t)^{T} g(t, x, u)\right\} e\right] d t .
\end{aligned}
$$

Theorem 4.2 (Strong duality). Let $\left(x^{0}, u^{0}\right)$ be a properly efficient solution for $(C P)$ and be normal, and let the Fréchet derivative $Q^{\prime}$ be surjective. Then there exist $\lambda^{0}, z^{0}(t)$ and $y^{0}(t)$ such that $\left(x^{0}, u^{0}, z^{0}, y^{0}, \lambda^{0}\right) \in F$ and the two vector objective functionals are equal. Also, if the invexity hypotheses of Theorem 4.1 are satisfied for every $(x, u, z, y, \lambda) \in F$, then $\left(x^{0}, u^{0}, z^{0}, y^{0}, \lambda^{0}\right)$ is a properly efficient solution of $(W C D)$. 
Proof. Since $\left(x^{0}, u^{0}\right)$ is a properly efficient solution for $(\mathrm{CP})$, it is also efficient. Therefore, by Theorem 3.4, there exist $\lambda^{0} \in \mathbb{R}^{p}$ and piecewise smooth functions $z^{0}: I \rightarrow \mathbb{R}^{n}$ and $y^{0}: I \rightarrow \mathbb{R}^{m}$ such that

$$
\begin{aligned}
& \begin{array}{l}
\lambda^{0^{T}} f_{x}\left(t, x^{0}, u^{0}\right)-z^{0}(t)^{T} h_{x}\left(t, x^{0}, u^{0}\right)-y^{0}(t)^{T} g_{x}\left(t, x^{0}, u^{0}\right)=\dot{z}^{0}(t), \\
t \in I,
\end{array} \\
& \begin{array}{l}
\lambda^{0^{T}} f_{u}\left(t, x^{0}, u^{0}\right)-z^{0}(t)^{T} h_{u}\left(t, x^{0}, u^{0}\right)-y^{0}(t)^{T} g_{u}\left(t, x^{0}, u^{0}\right)=0, \\
t \in I,
\end{array} \\
& \begin{array}{l}
y^{0}(t)^{T} g\left(t, x^{0}, u^{0}\right)=0, t \in I, \\
y^{0}(t) \geqq 0, t \in I, \\
\lambda^{0}>0, \quad \lambda^{0^{T}} e=1 .
\end{array}
\end{aligned}
$$

From (13), (14), (16) and (17), it follows that $\left(x^{0}, u^{0}, z^{0}, y^{0}, \lambda^{0}\right) \in F$. Since $\dot{x}^{0}(t)=h\left(t, x^{0}, u^{0}\right)$ and $y^{0}(t)^{T} g\left(t, x^{0}, u^{0}\right)=0$, the dual objective functional has the same value as the primal objective functional.

Now we claim that $\left(x^{0}, u^{0}, z^{0}, y^{0}, \lambda^{0}\right)$ is an efficient solution of (WCD). If not, then there exists $(\bar{x}, \bar{u}, \bar{z}, \bar{y}, \bar{\lambda}) \in F$ such that

$$
\begin{aligned}
& \int_{a}^{b}\left\{f^{r}(t, \bar{x}, \bar{u})-\bar{z}(t)^{T}(h(t, \bar{x}, \bar{u})-\dot{\bar{x}}(t))-\bar{y}(t)^{T} g(t, \bar{x}, \bar{u})\right\} d t \\
& >\int_{a}^{b} f^{r}\left(t, x^{0}, u^{0}\right) d t, \quad \text { for some } r \in\{1,2, \cdots, p\}, \text { and } \\
& \int_{a}^{b}\left\{f^{i}(t, \bar{x}, \bar{u})-\bar{z}(t)^{T}(h(t, \bar{x}, \bar{u})-\dot{\bar{x}}(t))-\bar{y}(t)^{T} g(t, \bar{x}, \bar{u})\right\} d t \\
& \geqq \int_{a}^{b} f^{i}\left(t, x^{0}, u^{0}\right) d t, \quad \text { for all } i \in\{1,2, \cdots, p\} \backslash\{r\} .
\end{aligned}
$$

The right hand side in the above inequalities contains only one term since $\dot{x}^{0}(t)=h\left(t, x^{0}, u^{0}\right)$ and $y^{0}(t)^{T} g\left(t, x^{0}, u^{0}\right)=0$. These inequalities contradict the conclusion of Theorem 4.1. Hence $\left(x^{0}, u^{0}, z^{0}, y^{0}, \lambda^{0}\right)$ is an efficient solution of (WCD).

Assume now that it is not a properly efficient solution of (WCD). Then there exist $(\bar{x}, \bar{u}, \bar{z}, \bar{y}, \bar{\lambda}) \in F$ and $i \in\{1,2, \cdots, p\}$ such that

$$
\begin{aligned}
& \int_{a}^{b}\left\{f^{i}(t, \bar{x}, \bar{u})-\bar{z}(t)^{T}(h(t, \bar{x}, \bar{u})-\dot{\bar{x}}(t))-\bar{y}(t)^{T} g(t, \bar{x}, \bar{u})\right\} d t \\
& >\int_{a}^{b} f^{i}\left(t, x^{0}, u^{0}\right) d t
\end{aligned}
$$


and

$$
\begin{aligned}
& \int_{a}^{b}\left\{f^{i}(t, \bar{x}, \bar{u})-\bar{z}(t)^{T}(h(t, \bar{x}, \bar{u})-\dot{\bar{x}}(t))-\bar{y}(t)^{T} g(t, \bar{x}, \bar{u})\right\} d t \\
& -\int_{a}^{b} f^{i}\left(t, x^{0}, u^{0}\right) d t>M\left[\int_{a}^{b} f^{j}\left(t, x^{0}, u^{0}\right) d t\right. \\
& \left.-\int_{a}^{b}\left\{f^{j}(t, \bar{x}, \bar{u})-\bar{z}(t)^{T}(h(t, \bar{x}, \bar{u})-\dot{\bar{x}}(t))-\bar{y}(t)^{T} g(t, \bar{x}, \bar{u})\right\} d t\right]
\end{aligned}
$$

for all $M>0$ and all $j \neq i$ satisfying

$$
\begin{aligned}
& \int_{a}^{b} f^{j}\left(t, x^{0}, u^{0}\right) d t>\int_{a}^{b}\left\{f^{j}(t, \bar{x}, \bar{u})-\bar{z}(t)^{T}(h(t, \bar{x}, \bar{u})-\dot{\bar{x}}(t))\right. \\
& \left.-\bar{y}(t)^{T} g(t, \bar{x}, \bar{u})\right\} d t .
\end{aligned}
$$

This means that

$$
\begin{aligned}
& \int_{a}^{b}\left\{f^{i}(t, \bar{x}, \bar{u})-\bar{z}(t)^{T}(h(t, \bar{x}, \bar{u})-\dot{\bar{x}}(t))-\bar{y}(t)^{T} g(t, \bar{x}, \bar{u})\right\} d t \\
& -\int_{a}^{b} f^{i}\left(t, x^{0}, u^{0}\right) d t
\end{aligned}
$$

can be made arbitrarily large, whereas

$$
\begin{aligned}
& \int_{a}^{b} f^{j}\left(t, x^{0}, u^{0}\right) d t-\int_{a}^{b}\left\{f^{j}(t, \bar{x}, \bar{u})-\bar{z}(t)^{T}(h(t, \bar{x}, \bar{u})-\dot{\bar{x}}(t))\right. \\
& \left.-\bar{y}(t)^{T} g(t, \bar{x}, \bar{u})\right\} d t
\end{aligned}
$$

is finite for all $j \neq i$. Therefore,

$$
\begin{aligned}
& \bar{\lambda}^{i} \int_{a}^{b}\left[\left\{f^{i}(t, \bar{x}, \bar{u})-\bar{z}(t)^{T}(h(t, \bar{x}, \bar{u})-\dot{\bar{x}}(t))-\bar{y}(t)^{T} g(t, \bar{x}, \bar{u})\right\}\right. \\
& \left.-f^{i}\left(t, x^{0}, u^{0}\right)\right] d t>\sum_{j \neq i} \bar{\lambda}^{j} \int_{a}^{b}\left[f^{j}\left(t, x^{0}, u^{0}\right)\right. \\
& \left.-\left\{f^{j}(t, \bar{x}, \bar{u})-\bar{z}(t)^{T}(h(t, \bar{x}, \bar{u})-\dot{\bar{x}}(t))-\bar{y}(t)^{T} g(t, \bar{x}, \bar{u})\right\}\right] d t,
\end{aligned}
$$

or

$$
\begin{aligned}
& \bar{\lambda}^{T} \int_{a}^{b}\left[f(t, \bar{x}, \bar{u})-\left(\bar{z}(t)^{T}(h(t, \bar{x}, \bar{u})-\dot{\bar{x}}(t))+\bar{y}(t)^{T} g(t, \bar{x}, \bar{u})\right) e\right] d t \\
& >\bar{\lambda}^{T} \int_{a}^{b} f\left(t, x^{0}, u^{0}\right) d t .
\end{aligned}
$$

This contradicts inequality (12). Hence $\left(x^{0}, u^{0}, z^{0}, y^{0}, \lambda^{0}\right)$ is a properly efficient solution of (WCD). 
For validating a converse duality theorem, we may rewrite the problem (WCD) in minimization form as follows:

Minimize $\quad \psi(x, u(t), z(t), y(t), \lambda) \quad$ subject to

$$
\begin{gathered}
x(a)=\alpha, \quad x(b)=\beta, \\
\psi_{1}(t, x(t), u(t), z(t), \dot{z}(t), y(t), \lambda)=0, t \in I, \\
\psi_{2}(t, x(t), u(t), z(t), y(t), \lambda)=0, t \in I, \\
y(t) \geqq 0, t \in I, \\
\lambda>0, \quad \lambda^{T} e=1,
\end{gathered}
$$

where

$$
\begin{aligned}
& \begin{aligned}
\psi(t, x, u, z, y, \lambda)= & \int_{a}^{b}\left[f(t, x, u)-\left\{z(t)^{T}(h(t, x, u)-\dot{x}(t))\right.\right. \\
& \left.\left.+y(t)^{T} g(t, x, u)\right\} e\right] d t
\end{aligned} \\
& \psi_{1}(t, x, u, z, \dot{z}, y, \lambda)=\lambda^{T} f_{x}(t, x, u)-z(t)^{T} h_{x}(t, x, u)-y(t)^{T} g_{x}(t, x, u)-\dot{z}(t)
\end{aligned}
$$

and

$$
\psi_{2}(t, x, u, z, y, \lambda)=\lambda^{T} f_{u}(t, x, u)-z(t)^{T} h_{u}(t, x, u)-y(t)^{T} g_{u}(t, x, u) .
$$

Consider $\psi_{1}(\cdot, x(\cdot), u(\cdot), z(\cdot), \dot{z}(\cdot), y(\cdot), \lambda)$ as defining a mapping $Q^{(1)}: X \times$ $U \times Z \times Y \times \mathbb{R}^{p} \rightarrow B_{1}$, where

(i) $Z$ is the space of piecewise smooth functions $z: I \rightarrow \mathbb{R}^{n}$,

(ii) $Y$ is the space of piecewise smooth functions $y: I \rightarrow \mathbb{R}^{m}$, and (iii) $B_{1}$ is a Banach space.

Also consider $\psi_{2}(\cdot, x(\cdot), u(\cdot), z(\cdot), y(\cdot), \lambda)$ as defining a mapping $Q^{(2)}: X \times$ $U \times Z \times Y \times \mathbb{R}^{p} \rightarrow B_{2}$, where $B_{2}$ is another Banach space. In order to apply Theorem 3.2 to (WCD), some restrictions are required on the equality constraints $\psi_{1}(\cdot)=0$ and $\psi_{2}(\cdot)=0$. It suffices if the Fréchet derivatives,

$$
\begin{aligned}
Q^{\prime(1)}= & {\left[Q_{x}{ }^{(1)}\left(x^{0}, u^{0}, z^{0}, y^{0}, \lambda^{0}\right), Q_{u}{ }^{(1)}\left(x^{0}, u^{0}, z^{0}, y^{0}, \lambda^{0}\right),\right.} \\
& Q_{z}^{(1)}\left(x^{0}, u^{0}, z^{0}, y^{0}, \lambda^{0}\right), Q_{y}{ }^{(1)}\left(x^{0}, u^{0}, z^{0}, y^{0}, \lambda^{0}\right), \\
& \left.Q_{\lambda}^{(1)}\left(x^{0}, u^{0}, z^{0}, y^{0}, \lambda^{0}\right)\right]
\end{aligned}
$$

and

$$
\begin{aligned}
Q^{\prime(2)}= & {\left[Q_{x}{ }^{(2)}\left(x^{0}, u^{0}, z^{0}, y^{0}, \lambda^{0}\right), Q_{u}{ }^{(2)}\left(x^{0}, u^{0}, z^{0}, y^{0}, \lambda^{0}\right),\right.} \\
& Q_{z}{ }^{(2)}\left(x^{0}, u^{0}, z^{0}, y^{0}, \lambda^{0}\right), Q_{y}{ }^{(2)}\left(x^{0}, u^{0}, z^{0}, y^{0}, \lambda^{0}\right), \\
& \left.Q_{\lambda}{ }^{(2)}\left(x^{0}, u^{0}, z^{0}, y^{0}, \lambda^{0}\right)\right]
\end{aligned}
$$

have weak* closed range. 
Theorem 4.3 (Converse duality). Let $\left(x^{0}, u^{0}, z^{0}, y^{0}, \lambda^{0}\right)$ be a properly efficient solution of $(W C D)$ and let

(H1): the Fréchet derivatives $Q^{\prime(1)}$ and $Q^{\prime(2)}$ have weak ${ }^{*}$ closed range, and (H2): the matrix

$$
\left[\begin{array}{ll}
\lambda^{0^{T}} f_{x x}-z^{0}(t)^{T} h_{x x}-y^{0}(t)^{T} g_{x x}, & \lambda^{0^{T}} f_{u x}-z^{0}(t)^{T} h_{u x}-y^{0}(t)^{T} g_{u x} \\
\lambda^{0^{T}} f_{x u}-z^{0}(t)^{T} h_{x u}-y^{0}(t)^{T} g_{x u}, & \lambda^{0^{T}} f_{u u}-z^{0}(t)^{T} h_{u u}-y^{0}(t)^{T} g_{u u}
\end{array}\right]
$$

be positive or negative definite for all $t \in I$. Then $\left(x^{0}, u^{0}\right) \in K$ and the two objective values are equal. Also, if the invexity hypotheses of Theorem 4.1 are satisfied for $\left(x^{0}, u^{0}, z^{0}, y^{0}, \lambda^{0}\right) \in F$, then $\left(x^{0}, u^{0}\right)$ is a properly efficient solution of $(C P)$.

Proof. Since $\left(x^{0}, u^{0}, z^{0}, y^{0}, \lambda^{0}\right)$ is a properly efficient solution of (WCD), by Theorem 3.2, there exist $\xi \in \mathbb{R}^{p}, \theta \in \mathbb{R}_{+}^{p}, \eta \in \mathbb{R}$, and piecewise smooth functions $\beta: I \rightarrow \mathbb{R}^{n}, \gamma: I \rightarrow \mathbb{R}^{r}$ and $\delta: I \rightarrow \mathbb{R}^{m}$ such that

$$
\begin{aligned}
& \xi^{T} f_{x}-\left(\xi^{T} e\right)\left\{z^{0}(t)^{T} h_{x}+y^{0}(t)^{T} g_{x}+\dot{z}^{0}(t)\right\} \\
& -\beta(t)^{T}\left\{\lambda^{0^{T}} f_{x x}-z^{0}(t)^{T} h_{x x}-y^{0}(t)^{T} g_{x x}\right\} \\
& -\gamma(t)^{T}\left\{\lambda^{0^{T}} f_{u x}-z^{0}(t)^{T} h_{u x}-y^{0}(t)^{T} g_{u x}\right\}=0, t \in I, \\
& \xi^{T} f_{u}-\left(\xi^{T} e\right)\left\{z^{0}(t)^{T} h_{u}+y^{0}(t)^{T} g_{u}\right\} \\
& -\beta(t)^{T}\left\{\lambda^{0^{T}} f_{x u}-z^{0}(t)^{T} h_{x u}-y^{0}(t)^{T} g_{x u}\right\} \\
& -\gamma(t)^{T}\left\{\lambda^{0^{T}} f_{u u}-z^{0}(t)^{T} h_{u u}-y^{0}(t)^{T} g_{u u}\right\}=0, t \in I, \\
& \left(\xi^{T} e\right)\left(h-\dot{x}^{0}\right)-\beta(t)^{T} h_{x}+\beta(t)-\gamma(t)^{T} h_{u}=0, t \in I, \\
& \left(\xi^{T} e\right) g-\beta(t)^{T} g_{x}-\gamma(t)^{T} g_{u}-\delta(t)=0, t \in I, \\
& \beta(t)^{T} f_{x}^{i}+\gamma(t)^{T} f_{u}^{i}+\theta^{i}+\eta=0, t \in I, i \in\{1,2, \cdots, p\} \\
& \delta(t)^{T} y^{0}(t)=0, t \in I, \\
& \theta^{T} \lambda^{0}=0 \\
& (\xi, \theta, \delta(t)) \geqq 0,(\xi, \theta, \eta, \beta(t), \gamma(t), \delta(t)) \neq 0, t \in I .
\end{aligned}
$$

The relations (18) with (8), and (19) with (9) respectively yield

$$
\begin{aligned}
& \left(\xi-\left(\xi^{T} e\right) \lambda^{0}\right)^{T} f_{x}-\beta(t)^{T}\left\{\lambda^{0^{T}} f_{x x}-z^{0}(t)^{T} h_{x x}-y^{0}(t)^{T} g_{x x}\right\} \\
& -\gamma(t)^{T}\left\{\lambda^{0^{T}} f_{u x}-z^{0}(t)^{T} h_{u x}-y^{0}(t)^{T} g_{u x}\right\}=0, t \in I
\end{aligned}
$$


and

$$
\begin{aligned}
& \left(\xi-\left(\xi^{T} e\right) \lambda^{0}\right)^{T} f_{u}-\beta(t)^{T}\left\{\lambda^{0^{T}} f_{x u}-z^{0}(t)^{T} h_{x u}-y^{0}(t)^{T} g_{x u}\right\} \\
& -\gamma(t)^{T}\left\{\lambda^{0^{T}} f_{u u}-z^{0}(t)^{T} h_{u u}-y^{0}(t)^{T} g_{u u}\right\}=0, t \in I .
\end{aligned}
$$

The equations (26) and (27) can now be written in the matrix form:

$$
\begin{aligned}
& {\left[\begin{array}{l}
f_{x}^{T}\left(\xi-\left(\xi^{T} e\right) \lambda^{0}\right) \\
f_{u}^{T}\left(\xi-\left(\xi^{T} e\right) \lambda^{0}\right)
\end{array}\right]} \\
& -\left[\begin{array}{ll}
\lambda^{0^{T}} f_{x x}-z^{0}(t)^{T} h_{x x}-y^{0}(t)^{T} g_{x x}, & \lambda^{0^{T}} f_{u x}-z^{0}(t)^{T} h_{u x}-y^{0}(t)^{T} g_{u x} \\
\lambda^{0^{T}} f_{x u}-z^{0}(t)^{T} h_{x u}-y^{0}(t)^{T} g_{x u}, & \lambda^{0^{T}} f_{u u}-z^{0}(t)^{T} h_{u u}-y^{0}(t)^{T} g_{u u}
\end{array}\right] \\
& \times\left[\begin{array}{l}
\beta(t) \\
\gamma(t)
\end{array}\right]=0, t \in I .
\end{aligned}
$$

The relation (24), because of $\theta \geqq 0$ and $\lambda^{0}>0$ implies $\theta=0$.

Multiplying (22) by $\left(\xi^{i}-\left(\xi^{T} e\right) \lambda^{0^{i}}\right)$ and summing over $i=1,2, \cdots, p$, we have

$$
\begin{aligned}
& \beta(t)^{T} \sum_{i=1}^{p} f_{x}^{i}\left(\xi^{i}-\left(\xi^{T} e\right) \lambda^{0^{i}}\right)+\gamma(t)^{T} \sum_{i=1}^{p} f_{u}^{i}\left(\xi^{i}-\left(\xi^{T} e\right) \lambda^{0^{i}}\right) \\
& +\eta \sum_{i=1}^{p}\left(\xi^{i}-\left(\xi^{T} e\right) \lambda^{0^{i}}\right)=0
\end{aligned}
$$

or

$$
\beta(t)^{T} f_{x}^{T}\left(\xi-\left(\xi^{T} e\right) \lambda^{0}\right)+\gamma(t)^{T} f_{u}^{T}\left(\xi-\left(\xi^{T} e\right) \lambda^{0}\right)=0 \quad \text { using (11) }
$$

which can be written as

$$
\left[\begin{array}{l}
\beta(t) \\
\gamma(t)
\end{array}\right]^{T}\left[\begin{array}{l}
f_{x}^{T}\left(\xi-\left(\xi^{T} e\right) \lambda^{0}\right) \\
f_{u}^{T}\left(\xi-\left(\xi^{T} e\right) \lambda^{0}\right)
\end{array}\right]=0, t \in I .
$$

Multiplying (28) by $\left(\beta(t)^{T}, \gamma(t)^{T}\right)$ and then using (29), we have

$$
\begin{aligned}
& {\left[\begin{array}{l}
\beta(t) \\
\gamma(t)
\end{array}\right]^{T}} \\
& \times\left[\begin{array}{ll}
\lambda^{0^{T}} f_{x x}-z^{0}(t)^{T} h_{x x}-y^{0}(t)^{T} g_{x x}, & \lambda^{0^{T}} f_{u x}-z^{0}(t)^{T} h_{u x}-y^{0}(t)^{T} g_{u x} \\
\lambda^{0^{T}} f_{x u}-z^{0}(t)^{T} h_{x u}-y^{0}(t)^{T} g_{x u}, & \lambda^{0^{T}} f_{u u}-z^{0}(t)^{T} h_{u u}-y^{0}(t)^{T} g_{u u}
\end{array}\right] \\
& \times\left[\begin{array}{l}
\beta(t) \\
\gamma(t)
\end{array}\right]=0, t \in I \text {. }
\end{aligned}
$$


This, in view of the hypothesis (H2) implies

$$
\beta(t)=0=\gamma(t), t \in I .
$$

Therefore, equations (20), (21) and (22) respectively yield

$$
\begin{gathered}
\left(\xi^{T} e\right)\left(h\left(t, x^{0}, u^{0}\right)-\dot{x}^{0}(t)\right)=0, t \in I, \\
\left(\xi^{T} e\right) g\left(t, x^{0}, u^{0}\right)-\delta(t)=0, t \in I,
\end{gathered}
$$

and

$$
\eta=0 .
$$

Now suppose $\xi=0$, then from (32), we get $\delta(t)=0, t \in I$. Thus

$$
(\xi, \theta, \eta, \beta(t), \gamma(t), \delta(t))=0, t \in I,
$$

a contradiction to (25). Hence $\xi \geq 0$ and therefore

$$
\xi^{T} e>0 \text {. }
$$

Using (33), equations (31) and (32) respectively give

$$
\dot{x}^{0}(t)=h\left(t, x^{0}, u^{0}\right), t \in I,
$$

and

$$
g\left(t, x^{0}, u^{0}\right)=\frac{\delta(t)}{\left(\xi^{T} e\right)} \geqq 0, t \in I .
$$

Equation (23) and the equality in (35) imply

$$
y^{0}(t)^{T} g\left(t, x^{0}, u^{0}\right)=0, t \in I .
$$

From (34) and (35), we have $\left(x^{0}, u^{0}\right) \in K$. Also, in view of (34) and (36), the two objective values are equal.

Now assume that $\left(x^{0}, u^{0}\right)$ is not efficient for $(\mathrm{CP})$. Then there exists $(\hat{x}, \hat{u}) \in K$ such that

$$
\int_{a}^{b} f(t, \hat{x}, \hat{u}) d t \leq \int_{a}^{b} f\left(t, x^{0}, u^{0}\right) d t .
$$

Using (34) and (36), we get

$$
\begin{aligned}
& \int_{a}^{b} f(t, \hat{x}, \hat{u}) d t \\
& \leq \int_{a}^{b}\left[f\left(t, x^{0}, u^{0}\right) d t-\left\{z^{0}(t)^{T}\left(h\left(t, x^{0}, u^{0}\right)-\dot{x}^{0}\right)+y^{0}(t)^{T} g\left(t, x^{0}, u^{0}\right)\right\} e\right] d t,
\end{aligned}
$$

a contradiction to the weak duality theorem. Hence $\left(x^{0}, u^{0}\right)$ is efficient.

If $\left(x^{0}, u^{0}\right)$ is improperly efficient, then there exists a point $(\hat{x}, \hat{u}) \in K$ and an $i \in\{1,2, \cdots, p\}$ such that

$$
\int_{a}^{b} f^{i}(t, \hat{x}, \hat{u}) d t<\int_{a}^{b} f^{i}\left(t, x^{0}, u^{0}\right) d t
$$


and

$$
\begin{aligned}
& {\left[\int_{a}^{b} f^{i}(t, \hat{x}, \hat{u}) d t-\int_{a}^{b} f^{i}\left(t, x^{0}, u^{0}\right) d t\right]} \\
& <M\left[\int_{a}^{b} f^{j}\left(t, x^{0}, u^{0}\right) d t-\int_{a}^{b} f^{j}(t, \hat{x}, \hat{u}) d t\right],
\end{aligned}
$$

for all $M>0$ and all $j \neq i$ satisfying

$$
\int_{a}^{b} f^{j}\left(t, x^{0}, u^{0}\right) d t<\int_{a}^{b} f^{j}(t, \hat{x}, \hat{u}) d t
$$

Similar to the proof in the last theorem, these inequalities yield,

$$
\lambda^{0^{T}} \int_{a}^{b} f(t, \hat{x}, \hat{u}) d t<\lambda^{0^{T}} \int_{a}^{b} f\left(t, x^{0}, u^{0}\right) d t
$$

which, in view of equations (34) and (36) contradicts inequality (12). Hence $\left(x^{0}, u^{0}\right)$ is a properly efficient solution of $(\mathrm{CP})$.

\section{Multiobjective control problem with free boundary conditions}

The above results may also be applied to the multiobjective control problem with free boundary conditions. If the targets $x(a)$ and $x(b)$ are not restricted, we obtain the following primal problem:

(CPF): Minimize $\int_{a}^{b} f(t, x, u) d t \quad$ subject to

$$
\begin{gathered}
\dot{x}(t)=h(t, x, u), t \in I, \\
g(t, x, u) \geqq 0, t \in I .
\end{gathered}
$$

The following dual (WCDF) to (CPF) includes the transversality condition $z(t)=0$, at $t=a$ and $t=b$ as new constraints.

(WCDF): Maximize

$$
\int_{a}^{b}\left[f(t, x, u)-\left\{z(t)^{T}(h(t, x, u)-\dot{x}(t))+y(t)^{T} g(t, x, u)\right\} e\right] d t
$$

subject to

$$
\begin{aligned}
& \lambda^{T} f_{x}(t, x, u)-z(t)^{T} h_{x}(t, x, u)-y(t)^{T} g_{x}(t, x, u)=\dot{z}(t), t \in I, \\
& \lambda^{T} f_{u}(t, x, u)-z(t)^{T} h_{u}(t, x, u)-y(t)^{T} g_{u}(t, x, u)=0, t \in I, \\
& y(t) \geqq 0, t \in I, \\
& \lambda>0, \\
& \lambda^{T} e=1, \\
& z(a)=0, \quad z(b)=0 .
\end{aligned}
$$


For these problems, the duality theorems of the preceding section can easily be validated. However, in Theoem 4.1, the term $\left.\eta^{T} z(t)\right|_{t=a} ^{t=b}$ vanishes using $z(a)=0=z(b)$ instead of the conditions $x(a)=\alpha=x^{0}(a)$ and $x(b)=\beta=x^{0}(b)$.

\section{Mond-Weir type duality}

The Mond-Weir type dual for $(\mathrm{CP})$ is as follows:

Mond-Weir Dual (MCD): $\quad$ Maximize $\quad \int_{a}^{b} f(t, x, u) d t \quad$ subject to

$$
\begin{aligned}
& x(a)=\alpha, \quad x(b)=\beta, \\
& \lambda^{T} f_{x}(t, x, u)-z(t)^{T} h_{x}(t, x, u)-y(t)^{T} g_{x}(t, x, u)=\dot{z}(t), t \in I, \\
& \lambda^{T} f_{u}(t, x, u)-z(t)^{T} h_{u}(t, x, u)-y(t)^{T} g_{u}(t, x, u)=0, t \in I, \\
& \int_{a}^{b}\left(z(t)^{T}(h(t, x, u)-\dot{x}(t))+y(t)^{T} g(t, x, u)\right) d t \leqq 0, t \in I, \\
& y(t) \geqq 0, t \in I, \\
& \lambda>0 .
\end{aligned}
$$

Denoting by $G$ the set of feasible solutions of (MCD), we state the following Theorems 6.1-6.3 that can be proved as for Wolfe's type dual.

Theorem 6.1 (Weak duality). Let $\left(x^{0}, u^{0}\right) \in K$ and $(x, u, z, y, \lambda) \in G$. Assume that $\int_{a}^{b} \lambda^{T} f d t$ is pseudo-invex and $\int_{a}^{b}\left(z(t)^{T}(h-\dot{x})+y(t)^{T} g\right) d t$ is quasi-invex with respect to the same $\eta$ and $\xi$. Then

$$
\int_{a}^{b} f\left(t, x^{0}, u^{0}\right) d t \not \leq \int_{a}^{b} f(t, x, u) d t .
$$

Theorem 6.2 (Strong duality). Let $\left(x^{0}, u^{0}\right)$ be a properly efficient solution for $(C P)$ and be normal, and that the Fréchet derivative $Q^{\prime}$ be surjective. Then there exist Lagrange multiplier $\lambda^{0}, z^{0}(t)$ and $y^{0}(t)$ such that $\left(x^{0}, u^{0}, z^{0}, y^{0}, \lambda^{0}\right) \in G$ and the vector objective functionals of $(C P)$ and $(M C D)$ are equal at these points. Also, if the invexity hypotheses of Theorem 6.1 are satisfied for every $(x, u, z, y, \lambda) \in G$, then $\left(x^{0}, u^{0}, z^{0}, y^{0}, \lambda^{0}\right)$ is a properly efficient solution of $(M C D)$.

Theorem 6.3 (Converse duality). Let $\left(x^{0}, u^{0}, z^{0}, y^{0}, \lambda^{0}\right)$ be a properly efficient solution of $(M C D)$. Assume that

(I1) the Fréchet derivatives $Q^{\prime(1)}$ and $Q^{\prime(2)}$ have weak* closed range, (I2) the set $\left\{f_{x}^{1}, \cdots, f_{x}^{p}\right\}$ or $\left\{f_{u}^{1}, \cdots, f_{u}^{p}\right\}$ is linearly independent, and 
(I3) the matrix

$$
\left[\begin{array}{ll}
\lambda^{0^{T}} f_{x x}-z^{0}(t)^{T} h_{x x}-y^{0}(t)^{T} g_{x x}, & \lambda^{0^{T}} f_{u x}-z^{0}(t)^{T} h_{u x}-y^{0}(t)^{T} g_{u x} \\
\lambda^{0^{T}} f_{x u}-z^{0}(t)^{T} h_{x u}-y^{0}(t)^{T} g_{x u}, & \lambda^{0^{T}} f_{u u}-z^{0}(t)^{T} h_{u u}-y^{0}(t)^{T} g_{u u}
\end{array}\right]
$$

is nonsingular for all $t \in I$. Then $\left(x^{0}, u^{0}\right) \in K$ and the two objective values are equal. Also, if the hypotheses of Theorem 6.1 are satisfied for $\left(x^{0}, u^{0}, z^{0}, y^{0}, \lambda^{0}\right) \in G$, then $\left(x^{0}, u^{0}\right)$ is a properly efficient solution of $(C P)$.

The duality results similar to Theorems 6.1-6.3 can also be established for the following pair of dual multiobjective control problems with free boundary conditions in view of the modifications described in the preceding section.

Primal (CPF): Minimize $\int_{a}^{b} f(t, x, u) d t \quad$ subject to

$$
\begin{gathered}
\dot{x}(t)=h(t, x, u), t \in I, \\
g(t, x, u) \geqq 0, t \in I .
\end{gathered}
$$

Dual (MCDF): Maximize $\quad \int_{a}^{b} f(t, x, u) d t \quad$ subject to

$$
\begin{aligned}
& \lambda^{T} f_{x}(t, x, u)-z(t)^{T} h_{x}(t, x, u)-y(t)^{T} g_{x}(t, x, u)=\dot{z}(t), t \in I, \\
& \lambda^{T} f_{u}(t, x, u)-z(t)^{T} h_{u}(t, x, u)-y(t)^{T} g_{u}(t, x, u)=0, t \in I, \\
& \int_{a}^{b}\left(z(t)^{T}(h(t, x, u)-\dot{x}(t))+y(t)^{T} g(t, x, u)\right) d t \leqq 0, t \in I, \\
& y(t) \geqq 0, t \in I, \\
& \lambda>0, \\
& z(a)=0, \quad z(b)=0 .
\end{aligned}
$$

\section{Mathematical programming}

If $f, h$ and $g$ are independent of $t$ and $u$, then (CP), (WCD) and (MCD) reduce the following problems:

Primal (SP): Minimize $f(x)$ subject to

$$
\begin{aligned}
& h(x)=0, \\
& g(x) \geqq 0 .
\end{aligned}
$$

Wolfe Dual (WSD): $\quad$ Maximize $f(x)-\left[z^{T} h(x)+y^{T} g(x)\right] e$ subject to

$$
\begin{aligned}
& \lambda^{T} f_{x}(x)-z^{T} h_{x}(x)-y^{T} g_{x}(x)=0, \\
& y \geqq 0,
\end{aligned}
$$




$$
\begin{aligned}
& \lambda>0, \\
& \lambda^{T} e=1 .
\end{aligned}
$$

Mond-Weir Dual (MSD): Maximize $f(x)$ subject to

$$
\begin{aligned}
& \lambda^{T} f_{x}(x)-z^{T} h_{x}(x)-y^{T} g_{x}(x)=0, \\
& z^{T} h(x)+y(t)^{T} g(x) \leqq 0, \\
& y \geqq 0, \\
& \lambda>0 .
\end{aligned}
$$

The optimality and duality for the above problems have been discussed in $[4,5]$.

\section{References}

[1] Chandra, S., Craven, B. D., Husain, I., A class of nondifferentiable control problems, J. Optim. Theory Appl. 56 (1988), 227-243.

[2] Craven, B. D., Mathematical Programming and Control Theory, Chapman and Hall, London, 1978.

[3] Geoffrion, A. M., Proper efficiency and the theory of vector maximization, J. Math. Anal. Appl. 22 (1968), 618-630.

[4] Gulati, T. R., Islam, M. A., Efficiency and proper efficiency in differentiable multiobjective programming, Ganit 9 (1989), 55-62.

[5] Gulati, T. R., Islam, M. A., Sufficiency and duality in multiobjective programming involving generalized F-convex functions, J. Math. Anal. Appl. 183 (1994), 181-195.

[6] Kanniappan, P., Necessary conditions for optimality of nondifferentiable convex multiobjective programming, J. Optim. Theory Appl. 40 (1983), 167-174.

[7] Mond, B., Hanson, M. A., Duality for control problems, SIAM J. Control 6 (1968), $114-120$.

[8] Mond, B., Smart, I., Duality and sufficiency in control problems with invexity, J. Math. Anal. Appl. 136 (1988), 325-333.

T.R. Gulati

Department of Mathematics InDian Institute of TECHNOLOGY ROORKEE-247 667

INDIA

EMAIL:TRGORFMA@IITR.ERNET.IN

A. Ahmed

Department of Statistics

UNIVERSITY OF KASHMIR

HazRATbal, SRinagar -190006

KASHMIR, INDIA

EMAIL:AQLSTAT@YAHOO.CO.IN
I. HUSAIN Department of Mathematics National Institute of Technology HazRatbal, SRINAGAR-190 006

KASHMIR, INDIA EMAIL: IHUSAIN11@YAHOO.COM 